Check for updates

Cite this: RSC Adv., 2019, 9, 1501

\title{
Unveiling the helicity switching mechanism of a rigid two-tiered stacked architecture $\dagger$
}

\begin{abstract}
Peng Liu, (D) * Yafei Duan, Xihui Bian (D) and Xiaoyao Tan
Conformational inversion of foldamers has been shown to transmit signals across the lipid membrane. Helicity switching is critical to fulfilling this function. Despite the importance of the conformational inversion, the mechanism that underlies the helicity switching process remains unclear. In the present contribution, a rigid two-tiered stacked architecture (2T) has been investigated the atomic level using molecular simulations. The architecture consists of two conjugated cores and three flexible side chains. Two- and three-dimensional free-energy landscapes characterizing the isomerization of $2 \mathrm{~T}$ reveal a four-stage helicity switching process. Four stages involve the flipping of three peripheral aromatic rings in the top tier and rotating of the bottom tier relative to the top one. The highest barrier hampering the transition between right-handed and left-handed helices emerges as the second benzene ring flips. Structural analysis shows that the ring flipping stretches the side chain, which leads to the deformation of conjugated cores, twist of dihedral angles within side chains, and the reorientation of amine moieties attached to chains. By deciphering the intricate mechanism whereby the rigid stacked architecture isomerizes, our understanding of the helicity switching is expected to be improved, helping in turn the construction of novel functional helices.
\end{abstract}

Received 8th November 2018

Accepted 3rd January 2019

DOI: $10.1039 / \mathrm{c} 8 \mathrm{ra09226e}$

rsc.li/rsc-advances
The property of helical architectures can be regulated by inducing a conformational inversion triggered by external stimuli, viz., high temperature, ${ }^{7}$ salt enrichment, ${ }^{8}$ and light irradiation. ${ }^{9}$ Inverting the helical direction can play an important role to transmit information across the lipid membrane. ${ }^{\mathbf{1 0}}$ To mimic the function of G-protein-coupled receptors, Clayden and co-workers designed and fabricated helical foldamers. ${ }^{11}$ Binding of a chiral ligand with one terminal site inverts $(P)$ - or/ and $(M)$-helix. The helicity inversion propagates along the full length of foldamers and carries information to a reporter located at another terminus. ${ }^{\mathbf{1 2}}$ Further experiments show that the helicity switching is critical for the communication between compartments in artificial tissues. ${ }^{13}$ However, the mechanism that underlies helicity switching is still an open question.

A two-tiered stacked architecture (2T) designed and fabricated by Hartley and co-workers ${ }^{\mathbf{1 4}}$ can be considered as a paradigm for helical structures. This architecture was constructed by assembling discotic cores functionalized with three arms through imine formation. Based on ${ }^{1} \mathrm{H}$ NMR spectra and DFT calculations, rotations of threes arms result in the helicity switching process (see Scheme 1A) occured in toluene. The freeenergy barrier of activation, $\Delta G^{\ddagger}$, was estimated as $c a$. $+7.0 \mathrm{kcal} \mathrm{mol}^{-1}$, assuming a negligible $\Delta S^{\ddagger}$. The primary thrust of the present contribution is identifying the switching pathway and understanding the underlying mechanism.

To the best of our knowledge, characterizing the helicity switching process within multi-tiered architecture at the molecular level is absent. To explore the unknown area, the 


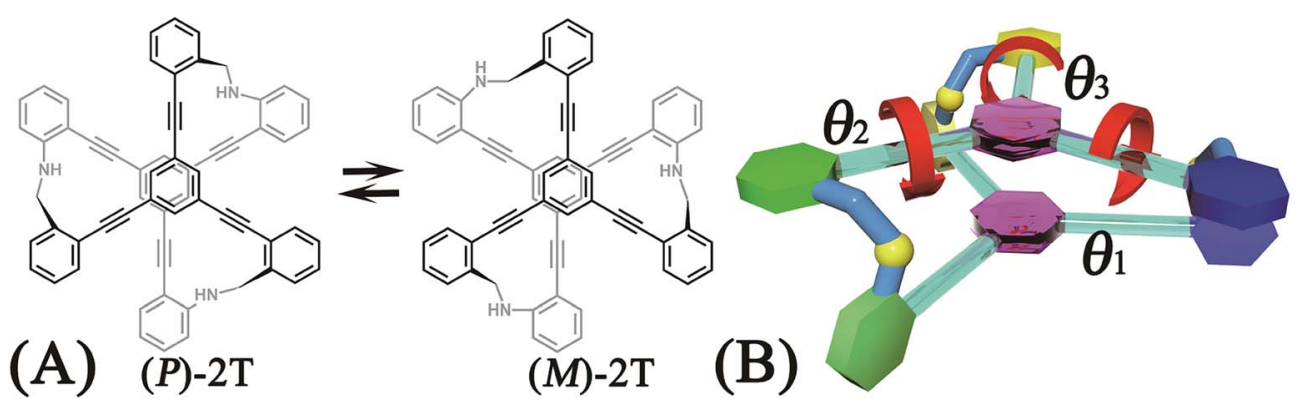

Scheme 1 (A) Chemical structure and the helicity switching process of the two-tiered stacked architecture (2T) studied in this contribution. (B) Definition of the reaction coordinate, $\theta_{1}, \theta_{2}$, and $\theta_{3}$, characterizing the rotational angle of the first (blue), second (green), and third (yellow) aromatic ring around alkyne bonds in the top tier, respectively.

three-dimensional free-energy landscape characterizing the rotational processes of three arms was determined. Intermediated states were identified and classified. All possible switching pathways share a four-stage pattern. These stages were characterized by drawing additional two- and threedimensional free-energy landscapes in further. Analysis of structural features together with the reconstruction of least freeenergy path help us to understand the helicity switching process of $2 \mathrm{~T}$.

\section{Simulation details}

\section{Molecular models}

The structure of $2 \mathrm{~T}$ presented in Scheme $1 \mathrm{~A}$ was constructed and immersed in a box of toluene. The initial size of the box was $70.0 \times 70.0 \times 69.3 \AA^{3}$ with 1728 toluene molecules. To improve the convergence of free-energy calculations, the top tier of $2 \mathrm{~T}$ was restrained to coplanar with the $x y$ plane by means of a harmonic potential with a force constant of $5.0 \mathrm{kcal} \mathrm{mol}^{-1}$ $\AA^{-1}$. In the subsequent molecular dynamics (MD) simulations, the spinning of the top tier around the $z$ axis was constrained. For the system, a $200 \mathrm{~ns}$ equilibrium MD simulation was preformed prior to the free-energy calculations.

\section{Molecular dynamics simulations}

All the MD simulations reported here were conducted employing the NAMD 2.12 program, ${ }^{15}$ with the CHARMM general force field. ${ }^{16,17}$ The temperature and pressure were maintained at $300.0 \mathrm{~K}$ and 1 bar, respectively, employing Langevin dynamics and the Langevin piston method. ${ }^{18}$ The length of the covalent bonds involving a hydrogen atom was frozen to its equilibrium value by means of the Shake/Rattle and Settle algorithms. ${ }^{19}$ The r-RESPA multiple-time-stepping algorithm was applied to integrate the equations of motion with a time step of 2 and $4 \mathrm{fs}$ for short- and long-range interactions, respectively. Short-range van der Waals and electrostatic interactions were truncated smoothly by means of a $12 \AA$ spherical cutoff with a switching function applied beyond $10 \AA$. The particle-mesh Ewald ${ }^{20}$ algorithm was used to compute the long-range electrostatic interactions with a mesh spacing of $<1.0 \AA$. Visualization and analysis of the MD trajectories were performed with VMD 1.9.3. ${ }^{21}$

\section{Free-energy calculations}

The free-energy landscapes in this contribution were generated using the extended adaptive biasing force (eABF) algorithm ${ }^{22-26}$ implemented within the COLVAR module ${ }^{27}$ of NAMD. ${ }^{28}$ Informed by MD simulations, the transition coordinate, either two- or three-dimensional, was formed by reaction coordinates $\theta_{1}, \theta_{2}$, and $\theta_{3}$, chosen as the spin angle of the first (blue), second (green), and third (yellow) aromatic unit in the top tier around the alkyne moieties, respectively (see Scheme 1B). Instantaneous values of the force were accrued in mesh grids. The variation of the free energy, $\Delta G$, was determined by integrating the average force acting on the transition coordinate.

In order to have a full view of the free-energy landscape characterizing the helicity switching process, a coarse grid was applied in the calculation of $\Delta G\left(\theta_{1}, \theta_{2}, \theta_{3}\right)$. For each spin angle, the full range of rotation $\left(-90^{\circ}\right.$ to $\left.270^{\circ}\right)$ was explored with a bin width of $6.0^{\circ}$. Further analysis reveals that 12 possible pathways of the helicity switching process share a common four-stage pattern containing three different kinds of stages, viz., stage I, stage II, and stage III. The first two stages were investigated by drawing the two-dimensional free-energy map along $\theta_{1}$ and $\theta_{2}$. These spin angles were explored in the range of rotation of $-90^{\circ}$ to $270^{\circ}$ with a bin width of $2.0^{\circ}$. The spin angle $\theta_{3}$ was confined in the range of $-90^{\circ}$ to $30^{\circ}$ by a harmonic wall restraint with a force constant of $5.0 \mathrm{kcal} \mathrm{mol}^{-1} \mathrm{deg}^{-1}$. The free-energy landscape describing stage III was determined by applying eABF method on the three transition coordinates $\theta_{1}, \theta_{2}$, and $\theta_{3}$. The transition pathway being explored spanned $60^{\circ} \leq \theta_{1} \leq 220^{\circ}, 60^{\circ} \leq \theta_{2} \leq$ $220^{\circ}$, and $-60^{\circ} \leq \theta_{3} \leq 120^{\circ}$. A bin width of $2.0^{\circ}$ was used for each transition coordinate. Total simulation time amounted to $1.0,1.0$, and $1.0 \mu \mathrm{s}$ for the helicity switching process, the first two and the third stages, respectively, representing an aggregate time of $3.0 \mu \mathrm{s}$. The least free-energy pathways connecting minima of the two- and three-dimensional free-energy landscapes were identified using the LFEP $^{29}$ and NMFEP ${ }^{30}$ algorithm. The concept of committor ${ }^{31,32}$ was utilized to prove 
that the model reaction coordinates formed by collective variable $\theta_{1}, \theta_{2}$ and $\theta_{3}$ correspond to an appropriate choice.

\section{Results and discussion}

\section{Free-energy landscape}

The three-dimensional free-energy landscape characterizing the helicity switching process of $2 \mathrm{~T}$ in toluene is depicted in Fig. 1A. 14 minima presented in the complete reaction coordinate space can be classified into six categories, viz., $(P)-2 \mathrm{~T}$, $(P)-2 \mathrm{~T}_{\underline{1 \mathrm{u}}},(P)-2 \mathrm{~T}_{\underline{2 \mathrm{u}}},(M)-2 \mathrm{~T},(M)-2 \mathrm{~T}_{\underline{1 \mathrm{u}}},(M)-2 \mathrm{~T}_{\underline{2 \mathrm{u}}}, \quad$ representing the $(P)$ - and $(M)$-conformations with 0,1 , and 2 aromatic unit(s) flipped, respectively. These minima are connected by 24 least freeenergy pathways. Based on these pathways, 12 possible helicity switching pathways can be proposed. Enumeration of every possible pathway can be found in the ESI (see Table S1†). Every switching pathway contains four stages, viz., $(P)-2 \mathrm{~T} \rightarrow$ $(P)-2 \mathrm{~T}_{\underline{1 \mathrm{u}}},(P)-2 \mathrm{~T}_{\underline{1 \mathrm{u}}} \rightarrow(P)-2 \mathrm{~T}_{\underline{2 u}},(P)-2 \mathrm{~T}_{\underline{2 u}} \rightarrow(M)-2 \mathrm{~T}_{\underline{1 \mathrm{u}}}$ and $(M)-2 \mathrm{~T}_{1 \mathrm{u}}$ $\rightarrow(M)-2 \mathrm{~T}$ or $(P)-2 \mathrm{~T} \rightarrow(P)-2 \mathrm{~T}_{\underline{1 \mathrm{u}}},(P)-2 \mathrm{~T}_{\underline{1 \mathrm{u}}} \rightarrow(M)-2 \mathrm{~T}_{\underline{2 \mathrm{u}}},(M)-2 \mathrm{~T}_{\underline{\underline{\mathrm{u}}}}$

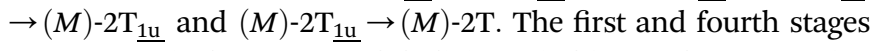
are symmetrical. Hence, each helicity switching pathway contains three different stages. In the following section, our attention was focused on the first three stage of the switching pathway $\quad(P)-2 \mathrm{~T}_{123} \rightarrow(P)-2 \mathrm{~T}_{123} \rightarrow(P)-2 \mathrm{~T}_{123} \rightarrow(M)-2 \mathrm{~T}_{12 \underline{3}} \rightarrow(M)-2 \mathrm{~T}_{123}$ (marked by the red arrow in Fig. 1B). Involved conformers can be found in Fig. 1C-G. To prove that the sampling in the $1.0 \mu \mathrm{s} \mathrm{eABF}$ calculations is ergodic, the distribution of the spin angle of the bottom tier relative to the top tier within $200 \mathrm{~ns}, 400 \mathrm{~ns}, 600 \mathrm{~ns}$, $800 \mathrm{~ns}$, and $1.0 \mu \mathrm{s}$ have been calculated, respectively (see Fig. S1 $\dagger$ ). Two peaks around $c a .-25^{\circ}$ and $25^{\circ}$ correspond to $(P)$ - and $(M)$ configurations. Increasing the sampling time only enhances the sampling density. The two-peak pattern shared by five curves remains unchanged. There results prove that the sampling is ergodic.

\section{Free-energy pathways}

To examine the first two stages of the helicity switching process in detail, a two-dimensional free-energy surface was generated along $\theta_{1}$ and $\theta_{2}$ with confined $\theta_{3}$ (see Fig. 2A). This map features four basins corresponding to $(P)-2 \mathrm{~T}_{123},(P)-2 \mathrm{~T}_{12 \underline{3}}$, $(P)-2 \mathrm{~T}_{123},(P)-2 \mathrm{~T}_{123}$. These basins are separated by four ridges. One gap is found on each ridge. The least free-energy pathways for $(P)-2 \mathrm{~T}_{123} \rightarrow(P)-2 \mathrm{~T}_{123}$ and $(P)-2 \mathrm{~T}_{\underline{123}} \rightarrow(P)-2 \mathrm{~T}_{\underline{12} 3}$ were determined using the LFEP algorithm.

To prove that the confinement on $\theta_{3}$ does not bias calculated results, the free-energy landscapes spanned by $\theta_{1}$ and $\theta_{2}$ of twoand three-dimensional eABF calculations were compared. For

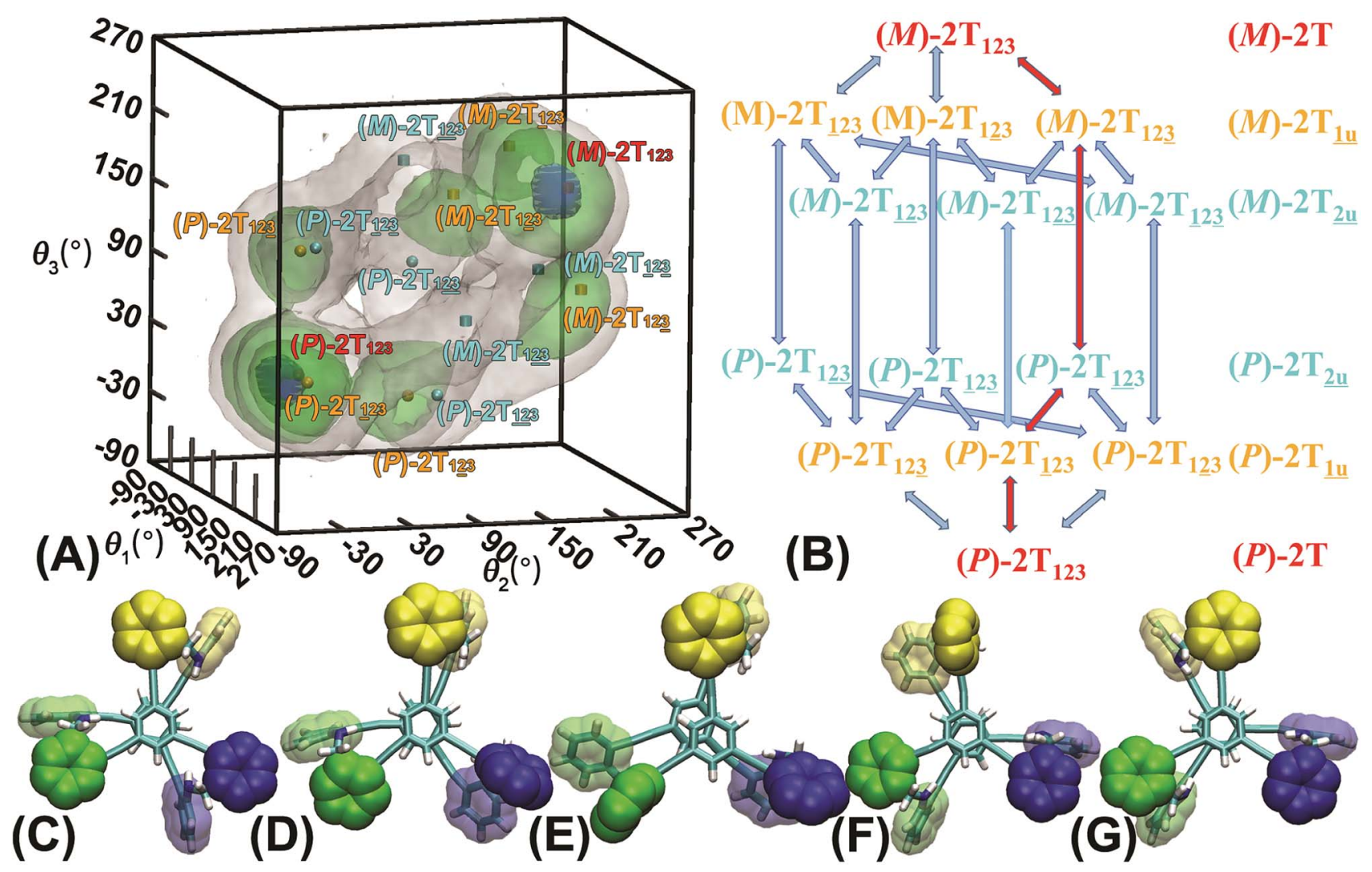

Fig. 1 (A) Three-dimensional free-energy landscape characterizing the helicity inversion process of 2T. Free-energy isosurfaces are shown for respective values of 1 (blue), 5 (green), $8 \mathrm{kcal} \mathrm{mol}^{-1}$ (white). Fourteen local minima are located in the landscape. (B) The scheme representing possible pathways connecting 14 local minima. One possible helicity inversion pathway is labeled by the red arrows. The involved conformers are shown as Licorice and VDW representation. Conformers are labeled by capital letters: (C) $(P)-2 \mathrm{~T}_{123},(\mathrm{D})(P)-2 \mathrm{~T}_{\underline{123}},(\mathrm{E})(P)-2 \mathrm{~T}_{\underline{12} 3},(\mathrm{~F})(M)-2 \mathrm{~T}_{12 \underline{3}}$, and (G) $(M)-2 T_{123}$. 

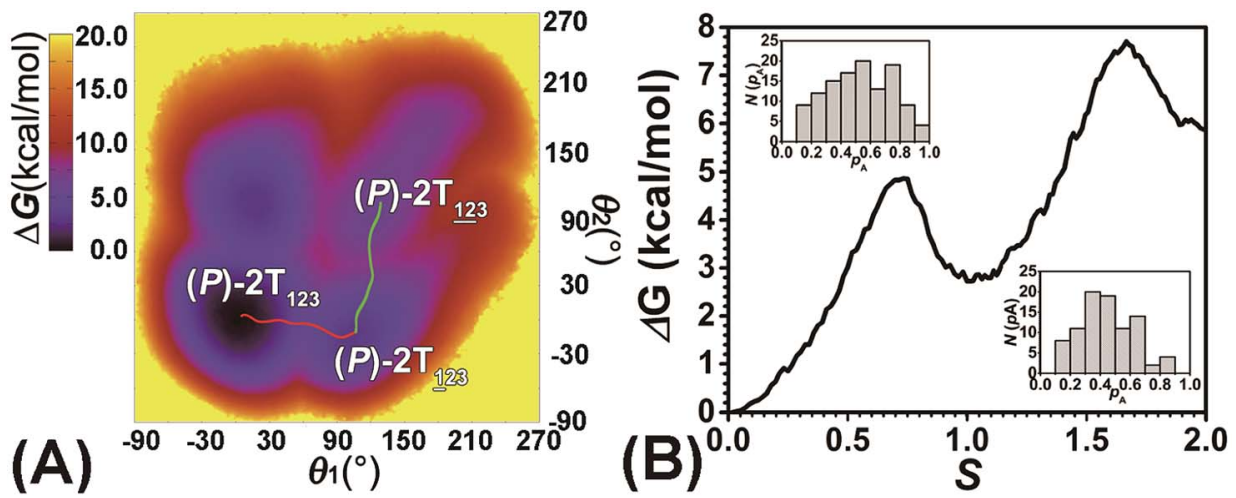

Fig. 2 (A) Free-energy surface characterizing the transition from $(P)-2 \mathrm{~T}_{123}$ to $(P)-2 \mathrm{~T}_{123}$ with a confined $\theta_{3}\left(-90^{\circ} \leq \theta_{3} \leq 30^{\circ}\right)$. (B) Free-energy profile as a function of the position $(s)$ along the least free-energy pathway connecting $(P)-2 \mathrm{~T}_{123}(s=0.0)$, $(P)-2 \mathrm{~T}_{123}(s=1.0)$, and $(P)-2 \mathrm{~T}_{123}(s=2.0)$. Inset: distributions of the committor, $p_{\mathrm{A}}$, at the positions near the saddle points around $\theta_{1}=61^{\circ}, \theta_{2}=3^{\circ}$ and around $\theta_{1}=121^{\circ}$, $\theta_{2}=55^{\circ}$ in the free-energy surface.

the three-dimensional case, the landscape spanned by $\theta_{1}$ and $\theta_{2}$ is calculated by integration over $\theta_{3}\left(-90^{\circ} \leq \theta_{3} \leq 30^{\circ}\right) .{ }^{33}$ Relevant results were gathered in Fig. S2. $\dagger$ Two landscapes are very similar. Least free-energy pathways within two landscapes were compared either. Two curves match well. These results prove that the constraint imposed on $\theta_{3}$ does not bias free-energy landscapes and least free-energy pathways.

The transition coordinates, $\theta_{1}$ and $\theta_{2}$, used herein, were checked by undertaking the committor analysis. Distributions of the committor, $p_{\mathrm{A}}$, at the position near the free-energy maxima, defined by $\left(\theta_{1}, \theta_{2}\right)$ near the saddle points, i.e., $\left(\theta_{1}=\right.$ $\left.61^{\circ}, \theta_{2}=3^{\circ}\right)$ and $\left(\theta_{1}=121^{\circ}, \theta_{2}=55^{\circ}\right)$, were determined (see Fig. 2B). The distributions are Gaussian-like and peaked at the probability of 0.5 . The transition coordinate, $\theta_{1}$ and $\theta_{2}$, represents a reasonable combination of the collective variables to describe the first two stages of the helicity switching process.

As the helicity switches, the first ring in the top tier rotates from a stable conformation $\left(-20^{\circ} \leq \theta \leq 20^{\circ}\right)$ to a quasistable one $\left(100^{\circ} \leq \theta \leq 120^{\circ}\right)$ in the first stage $\left(S_{0 \rightarrow 1}\right)$. Subsequent rotation of the second ring from the stable conformation to a quasistable one constitutes the second stage $\left(S_{1 \rightarrow 2}\right)$. Successively rotations of two peripheral rings in the top tier were detected (see Fig. S3A $\dagger$ ). Meanwhile, no obvious rotation of the bottom tier relative to the top tier was observed (see Fig. S3B $\dagger$ ). The free-energy profile for stage I and II as a function of the position $(s)$ is reported in Fig. 2B. $s$ is the order parameter of the pathway. The detailed definition can be found in the work of Branduardi et al. $.^{33} s=0.0, s=1.0$ and $s=2.0$ correspond to the global minimum $(P)-2 \mathrm{~T}_{123}\left(\theta_{1}=5^{\circ}, \theta_{2}=3^{\circ}, \Delta G_{s}=0.0=\right.$ $\left.0.0 \mathrm{kcal} \mathrm{mol}^{-1}\right)$ and two local minima $(P)-2 \mathrm{~T}_{123}\left(\theta_{1}=107^{\circ}, \theta_{2}=\right.$ $\left.-11^{\circ}, \Delta G_{s}=1.0=2.7 \mathrm{kcal} \mathrm{mol}^{-1}\right)$ and $(P)-2 \mathrm{~T}_{\underline{12} 3}\left(\theta_{1}=129^{\circ}, \theta_{2}=\right.$ $\left.103^{\circ}, \Delta G_{s}={ }_{2.0}=5.9 \mathrm{kcal} \mathrm{mol}^{-1}\right)$. Two peaks can be located in the free-energy profile. The height of the peaks relative to the global minimum, $\Delta G^{\ddagger}$, is estimated to be $4.9 \mathrm{kcal} \mathrm{mol}^{-1}$ in the stage $S_{0 \rightarrow 1}$ and $7.4 \mathrm{kcal} \mathrm{mol}^{-1}$ in the stages $S_{0 \rightarrow 2}$.

To examine stage III accurately, a three-dimensional freeenergy landscape was generated along $\theta_{1}, \theta_{2}$, and $\theta_{3}$ within shorter ranges and fine grids (see Fig. $3 \mathrm{~A}$ ). This landscape features an asymmetrical dumbbell. Two minima were located at terminal spheres. The least free-energy path from one minimum $(P)-2 \mathrm{~T}_{\underline{123}}\left(\theta_{1}=127^{\circ}, \theta_{2}=101^{\circ}, \theta_{3}=-15^{\circ}\right)$ to another $(M)-2 \mathrm{~T}_{12 \underline{3}}\left(\theta_{1}=171^{\circ}, \theta_{2}=205^{\circ}, \theta_{3}=85^{\circ}\right)$ was determined. Concomitant rotations of three peripheral rings in the top tier were detected (see Fig. S3C $\dagger$ ). The third peripheral ring in the top tier rotates from a stable conformation to the quasistable state. The first and second rings in the top tier transform from the quasistable state to another stable conformation $\left(160^{\circ} \leq \theta\right.$ $\leq 200^{\circ}$ ). Meanwhile, an obvious rotation of the bottom tier relative to the top tier around the $z$ axis indicates an adjustment of the framework for $2 \mathrm{~T}$ (see Fig. $\mathrm{S} 3 \mathrm{D} \dagger$ ).

To check the transition coordinate, $\theta_{1}, \theta_{2}$, and $\theta_{3}$, used herein, a committor analysis was undertaken. Distribution of the committor, $p_{\mathrm{A}}$, at the position near the free-energy maximum (around $\theta_{1}=131^{\circ}, \theta_{2}=113^{\circ}$, and $\theta_{3}=17^{\circ}$ ) was determined (see Fig. 3B). This distribution is Gaussian-like with a peak around $p_{\mathrm{A}}=0.5$. It suggests that the combination of collective variables, $\theta_{1}, \theta_{2}$, and $\theta_{3}$, is a reasonable transition coordinate to describe the third stage of the helicity switching pathway.

The free-energy profile for the third stage of the switching pathway as a function of $s$ is reported in Fig. 3B. $s=3.0$ corresponds to the minimum $(M)-2 \mathrm{~T}_{12 \underline{3}} \quad\left(\Delta G_{s}={ }_{3.0}=\Delta G_{s}=2.0\right.$ $\left.-3.7 \mathrm{kcal} \mathrm{mol}^{-1}=2.2 \mathrm{kcal} \mathrm{mol}^{-1}\right)$. This profile features a gentle slope with a week barrier emerges around the point ( $c a . s=2.3$ ). The height of the barrier, $\Delta G^{\ddagger}$, is estimated to be $0.6 \mathrm{kcal} \mathrm{mol}^{-1}$ in the stage $S_{2 \rightarrow 3}$ and $6.5 \mathrm{kcal} \mathrm{mol}^{-1}\left(\Delta G_{s}={ }_{2.0}+0.6 \mathrm{kcal} \mathrm{mol}^{-1}\right)$ in the stages $S_{0 \rightarrow 3}$.

Based on the observation in this section, a deeper understanding of the four-stage (helicity switching) process emerges. Helicity switching of $2 \mathrm{~T}$ involves rotations of three peripheral rings in the top tier from one stable conformation $\left(-20^{\circ} \leq \theta \leq\right.$ $\left.20^{\circ}\right)$ to a quasistable state $\left(100^{\circ} \leq \theta \leq 120^{\circ}\right)$, and then to another stable conformation $\left(160^{\circ} \leq \theta \leq 200^{\circ}\right)$. There is a peak in each stage. The highest peak appears in the second stage. The height of the peak relative to the global minimum, $\Delta G^{\ddagger}$, equal to $7.4 \mathrm{kcal} \mathrm{mol}^{-1}$, matches well with the experimental measurement. ${ }^{14}$ 


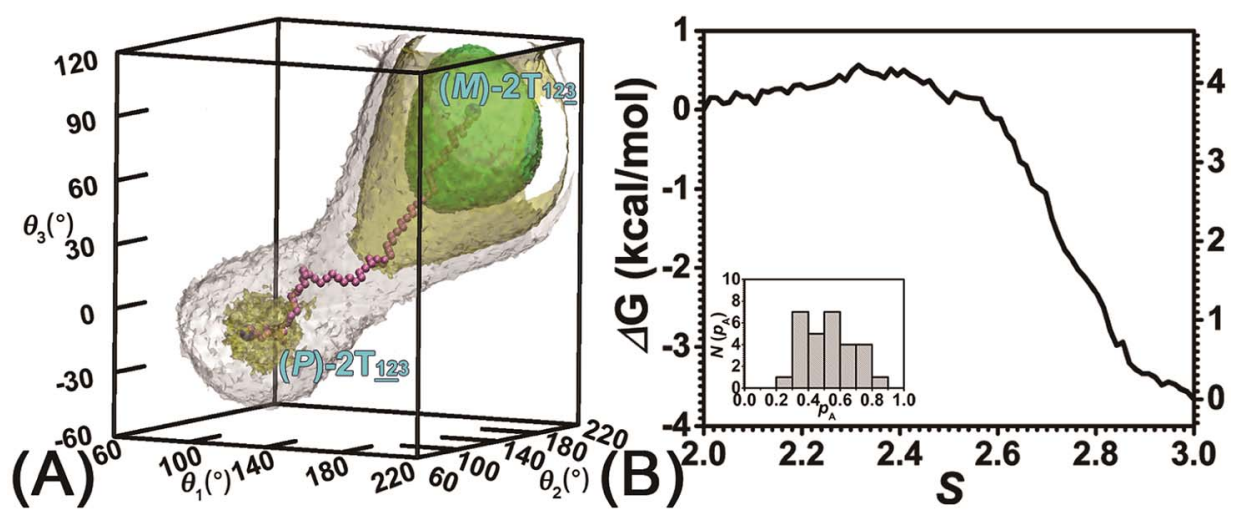

Fig. 3 (A) Three-dimensional free-energy landscape characterizing the transition from $(P)-2 \mathrm{~T}_{\underline{12} 3}$ to $(M)-2 \mathrm{~T}_{12 \underline{3}}$. Free-energy isosurfaces are shown for respective values of 2 (green), 4 (yellow), $5 \mathrm{kcal} \mathrm{mol}^{-1}$ (white). (B) Free-energy profile as a function of the position (s) along the least free-energy pathway. Inset: distribution of the committor, $p_{\mathrm{A}}$, at the positions near the peak point around $\left(\theta_{1}=131^{\circ}, \theta_{2}=113^{\circ}\right.$, and $\left.\theta_{3}=17^{\circ}\right)$.

\section{Interactions responsible for the helicity switching}

To identify the interaction components that contribute to the free-energy barrier, variation of constituent interactions of different nature was examined. 2T intramolecular (2T-self) interaction and 2T-solvent interaction were monitored along the least free-energy pathway. The resulting interaction components are gathered in Fig. $4 \mathrm{~A}$ and $\mathrm{B}$.

2T-Solvent. 2T-solvent term possesses three shallow valleys spanning $0.2 \leq s \leq 0.7,1.2 \leq s \leq 1.8$, and $2.2 \leq s \leq 2.8$ (with an average depth of ca. 2.0, 2.0, and $1.5 \mathrm{kcal} \mathrm{mol}^{-1}$, respectively), indicating a positive contribution to the helical switching process. To delve deeper into the structural origin of 2T-solvent term, the solvent accessible surface area of $2 \mathrm{~T}$ has been measured (see Fig. S4A and $\mathrm{B} \dagger$ ). The barrier in the middle range of each stage reveals an obvious increase of the contact area between $2 \mathrm{~T}$ and hydrophobic solvent molecules, resulting in a decrease of $2 \mathrm{~T}$-solvent interactions.

2T-Self. As illustrated in Fig. 4A and B, the 2T-self component features two high barriers in the first and second stages and an obvious slope in the third stage constituting the dominating contribution to the barriers and slope in the three stages of the least free-energy pathways. To delve into this component, the 2T-self term was reconstructed by combining the bonded and non-bonded interactions (van der Waals and electrostatic interactions) for cores and side chains. Every core contains three alkyne units attached to the same central aromatic unit.
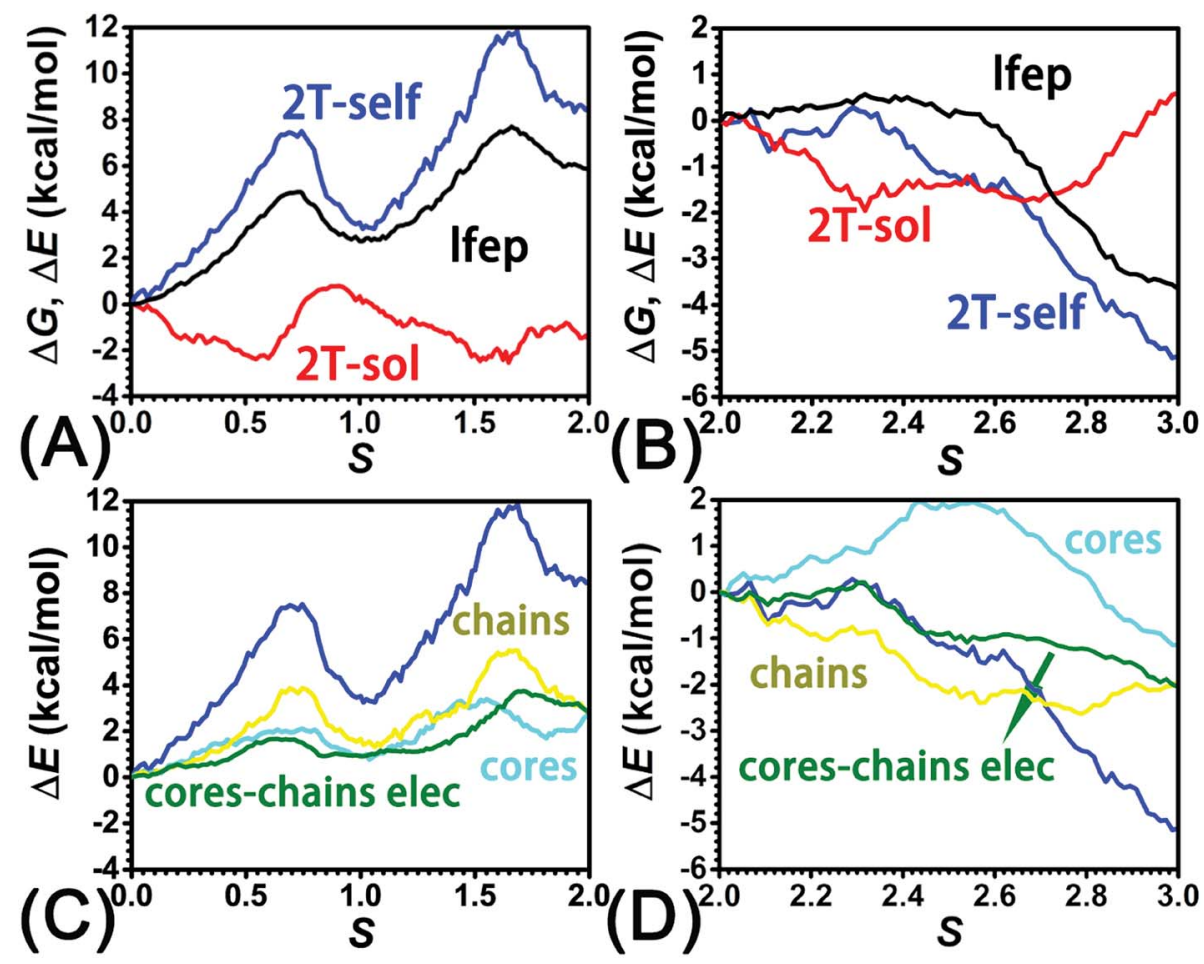

Fig. 4 (A) Reconstruction of the least free-energy pathway by combining 2T-solvent and 2T-self contributions in (A) stage I, II, and (B) III. Decomposition of 2T-self term into chains, cores, and cores-chains electrostatic terms in (C) stage I, II, and (D) III. 

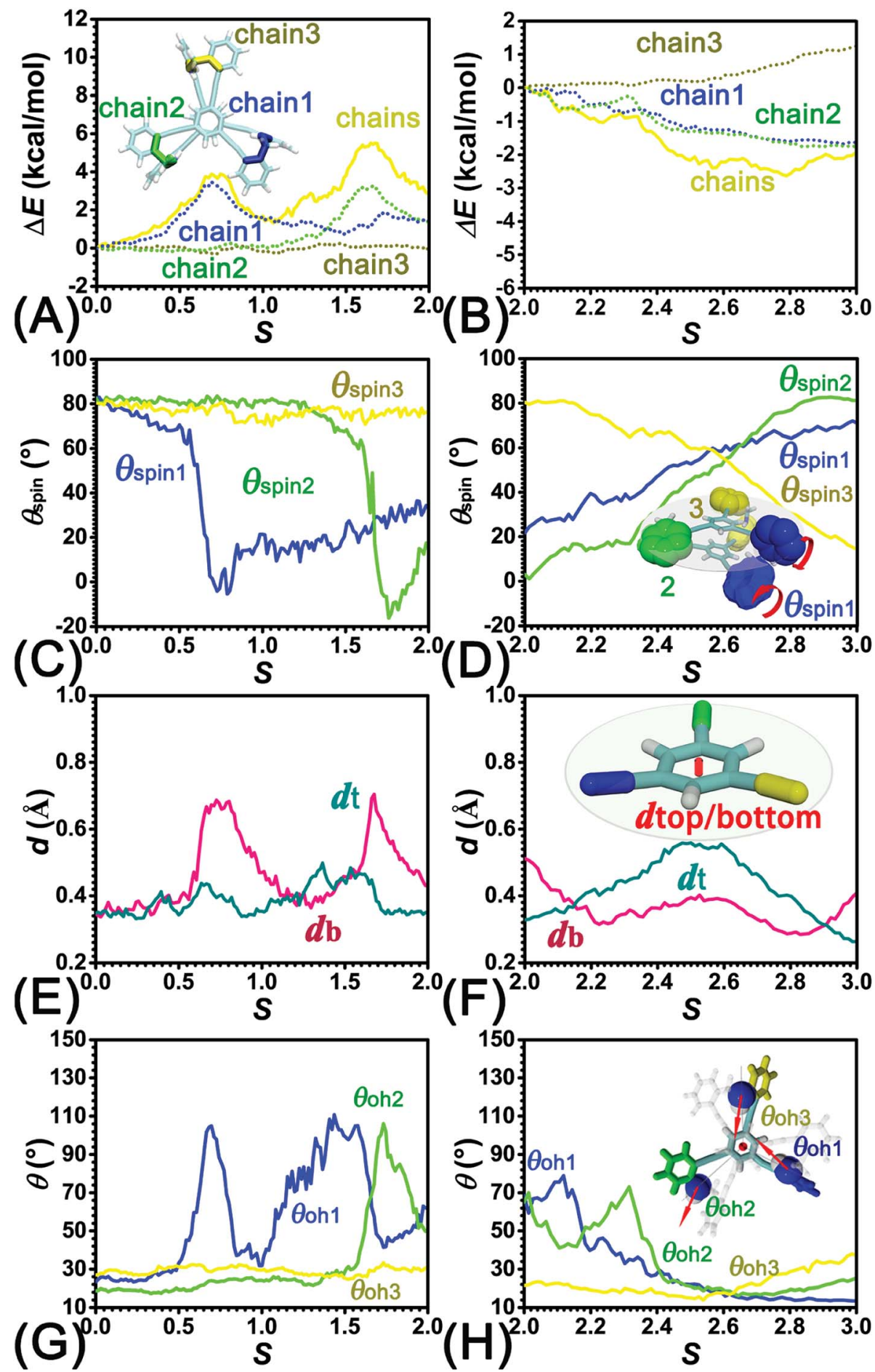

Fig. 5 Decomposition of the chains term into chain1, chain2, chain3 interactions in (A) stage I, II, and (B) III. Evolution of the average spin angles of aromatic rings within the bottom tier around the alkyne bonds in (C) stage I, II, and (D) III. The variation of average distances between the COM of the central benzene moiety and the plane spanned by the three alkyne units within the same tier in (E) stage I, II, and (F) III. Evolution of $\theta_{\text {oh }}$ as a function of $s$, where $\theta_{\text {oh }}$ is the angle formed between the dipole moment of the amine moiety and vector that connects the nitrogen atom and the central benzene moiety, in $(\mathrm{G})$ stage I, II, and (H) III.

Each chain is composed by two benzene rings connected by a linker containing an amine moiety. The cores-chains van der Waals term is negligible (see Fig. S4C and $\mathrm{D}_{\dagger}^{\dagger}$ ). Three components, viz., (side) chains, cores, cores-chains electrostatic interactions, were gathered in Fig. 4C and D.
Chains term. The chains term possesses a barrier in stage I and II and a gentle slope in stage III. It indicates that the chains term hampers the helicity switching in the first two stages. In stage III, chains interaction slightly favors the helical inversion process of 2T. Additional analysis was carried out by 
partitioning the chains term into chain1, chain2, and chain3 terms. The corresponding components are depicted in Fig. 5A and $\mathrm{B}$. The chain 1 term and chain 2 term contribute predominantly to the barrier of chains term in stage I and II, respectively. In stage III, the decrease of chain1 and chain2 terms partially counterbalanced by the increase of chain 3 term results in the slope in chains term.

To delve deeper into this analysis, the spin angles of aromatic rings within the bottom tier around the connected alkyne units were measured. The evolution of the average spin angles along $s$ is shown in Fig. $5 \mathrm{C}$ and D. In stage I, the aromatic ring (in the bottom tier) in chain1 rotates from the perpendicular conformation $\left(\mathrm{ca} .80^{\circ}\right)$ to a horizontal conformation (around $0^{\circ}$ ). Rings in chain 1 are pushed away from each other (see Fig. S4E and $\mathrm{F}^{\dagger}$ ). The twist of dihedral angles within chain1 (see Fig. S5 $\dagger$ ) causes a barrier in chain1 and chains term. No rotation of rings in other chains was observed. In stage II, rotation of the benzene ring (within the bottom tier) in chain2 from $c a .80^{\circ}$ to $0^{\circ}$ results in the barrier in chain 2 term. Rings (in the bottom tier) in chain 1 and chain 3 maintain the horizontal and perpendicular conformations, respectively. In stage III, switching back to the perpendicular conformations for rings (in the bottom tier) in chain1 and chain2 releases dihedral distortions. Although the ring (in the bottom tier) in chain3 rotating to the horizontal conformation causes novel twist, the dihedral distortions within $2 \mathrm{~T}$ are partially released, thus rationalizing that chains term is favorable in stage III.

Cores term. As illustrated in Fig. 4C and D, a broad barrier arises in the middle of each stage. The barriers in cores term can be ascribe to the geometrical deformation of the cores section. To monitor the degree of deformation, the distance between the center of mass (COM) of the central benzene moiety and the plane spanned by alkyne units within the same tier was monitored. The evolution of average distances along the least free-energy path are shown in Fig. 5E and F. The pronounced peaks found at around 0.7 (pink curve), 1.7 (pink curve), and 2.5 (dark cyan curve) correspond to the increase of out-of-plane deviations for the central benzene units from the conjugated aromatic cores, which is caused by the separation of benzene rings within side chains driven by the concomitant rotation of rings (see Fig. $\mathrm{S} 4 \dagger$ ). The effect of such deformation partially constitutes the contribution to the barrier in the freeenergy profile of stage I and II (no stage III).

Cores-chains electrostatic. As depicted in Fig. 4C and D, the cores-chains electrostatic term features a barrier, a barrier, and a gentle slope in stage I, II, and III, respectively. It suggests that the cores-chains electrostatic term disfavors the first two stages and favors stage III. To delve deeper into this observation, the orientation of amine moieties was measured, as shown in Fig. 5G and H. In stage I, rotating the first aromatic ring (blue, in the top tier) is accompanied by the reorientation of the amine unit within chain1. As the second benzene ring (green, in the top tier) spins in stage II, amine moieties within chain2 and chain3 alert their orientation separately. Reorientation of polarized amine moieties is energetically unfavorable, causing the barrier in the cores-chains electrostatic term of Fig. 4C. In stage III, amine moieties within chain 2 and chain 3 return to its initial orientation in stage III, rationalizing the former observation that the cores-chains electrostatic interaction favors stage III.

\section{Conclusion}

In this contribution, the helicity switching process within a twotiered stacked architecture designed by Hartley and coworkers was investigated. Three-dimensional free-energy landscape characterizing the helicity switching was determined. The helical inversion process is constituted by four stages containing three different kinds. The four stages correspond to the spinning of three peripheral benzene rings in the top tier and the rotation of bottom tier relative the top one around the $z$ axis. The height of the highest free-energy barrier located in the second stage was estimated as high as $7.4 \mathrm{kcal} \mathrm{mol}^{-1}$ matching with experimental observations. Based on the geometrical analysis and calculation of interactions, a switching mechanism was proposed. Spinning peripheral rings in the top tier causes the concomitant rotation of connected rings within the bottom tier leading to the separation of rings in the same side chain. The separation induces the geometrical deformation of the connected conjugated cores section and twist of dihedral angles within side chains. The twist reorientates polarized amine moieties attached to side chains. These factors collectively shape the least free-energy pathway in each stage.

The present results provide a complete understanding of the helicity switching process, which is crucial for the rational design of novel functional helices. The effects of structural modifications on the function of helices can be evaluated in silico. Attention of synthesis should be focused on the candidates screened from a series of lead structures constructed from fragment libraries. The invested effort and cost can be dramatically decreased. Combination of experimental and computational efforts is expected along this line.

\section{Conflicts of interest}

There are no conflicts to declare.

\section{Acknowledgements}

This study is supported by National Natural Science Foundation of China (No. 21603160, 91745116, 21875184, and 51678409).

\section{References}

1 Y. Ferrand and I. Huc, Acc. Chem. Res., 2018, 51, 970-977.

2 E. Yashima, K. Maeda, H. Iida, Y. Furusho and K. Nagai, Chem. Rev., 2009, 109, 6102-6211.

3 M. Liu, L. Zhang and T. Wang, Chem. Rev., 2015, 115, 73047397.

4 H. Lu and N. Kobayashi, Chem. Rev., 2016, 116, 6184-6261.

5 D. J. van Dijken, J. M. Beierle, M. C. A. Stuart, W. Szymański, W. R. Browne and B. L. Feringa, Angew. Chem., Int. Ed., 2014, 53, 5073-5077. 
6 D. Taura, S. Hioki, J. Tanabe, N. Ousaka and E. Yashima, ACS Catal., 2016, 6, 4685-4689.

7 M. Peterca, M. R. Imam, C.-H. Ahn, V. S. K. Balagurusamy, D. A. Wilson, B. M. Rosen and V. Percec, J. Am. Chem. Soc., 2011, 133, 2311-2328.

8 R. Liu, H. Liu, X. Chen, M. Kirby, P. O. Brown and K. Zhao, Cell, 2001, 106, 309-318.

9 M. Mathews, R. S. Zola, S. Hurley, D.-K. Yang, T. J. White, T. J. Bunning and Q. Li, J. Am. Chem. Soc., 2010, 132, 18361-18366.

10 R. A. Brown, V. Diemer, S. J. Webb and J. Clayden, Nat. Chem., 2013, 5, 853-860.

11 M. De Poli, W. Zawodny, O. Quinonero, M. Lorch, S. J. Webb and J. Clayden, Science, 2016, 352, 575-580.

12 J. Solà, S. P. Fletcher, A. Castellanos and J. Clayden, Angew. Chem., Int. Ed., 2010, 49, 6836-6839.

13 F. G. A. Lister, B. A. F. Le Bailly, S. J. Webb and J. Clayden, Nat. Chem., 2017, 9, 420-425.

14 F. Ren, K. J. Day and C. S. Hartley, Angew. Chem., Int. Ed., 2016, 55, 8620-8623.

15 J. C. Phillips, R. Braun, W. Wang, J. Gumbart, E. Tajkhorshid, E. Villa, C. Chipot, R. D. Skeel, L. Kalé and K. Schulten, J. Comput. Chem., 2005, 26, 1781-1802.

16 K. Vanommeslaeghe, E. Hatcher, C. Acharya, S. Kundu, S. Zhong, J. Shim, E. Darian, O. Guvench, P. Lopes, I. Vorobyov and A. D. Mackerell, J. Comput. Chem., 2009, 31, 671-690.

17 W. Yu, X. He, K. Vanommeslaeghe and A. D. MacKerell, J. Comput. Chem., 2012, 33, 2451-2468.

18 S. E. Feller, Y. Zhang, R. W. Pastor and B. R. Brooks, J. Chem. Phys., 1995, 103, 4613-4621.
19 J.-P. Ryckaert, G. Ciccotti and H. J. C. Berendsen, J. Comput. Phys., 1977, 23, 327-341.

20 T. Darden, D. York and L. Pedersen, J. Chem. Phys., 1993, 98, 10089-10092.

21 W. Humphrey, A. Dalke and K. Schulten, J. Mol. Graphics, 1996, 14, 33-38.

22 H. Fu, X. Shao, C. Chipot and W. Cai, J. Chem. Theory Comput., 2016, 12, 3506-3513.

23 A. Lesage, T. Lelièvre, G. Stoltz and J. Hénin, J. Phys. Chem. B, 2017, 121, 3676-3685.

24 E. Darve, M. A. Wilson and A. Pohorille, Mol. Simul., 2002, 28, 113-144.

25 J. Hénin and C. Chipot, J. Chem. Phys., 2004, 121, 2904-2914. 26 H. Fu, X. Shao, C. Chipot and W. Cai, J. Chem. Theory Comput., 2017, 13, 1566-1576.

27 G. Fiorin, M. L. Klein and J. Hénin, Mol. Phys., 2013, 111, 3345-3362.

28 B. Ensing, A. Laio, M. Parrinello and M. L. Klein, J. Phys. Chem. B, 2005, 109, 6676-6687.

29 M. Moradi, G. Enkavi and E. Tajkhorshid, Nat. Commun., 2015, 6, 8393.

30 A. C. Pan, D. Sezer and B. Roux, J. Phys. Chem. B, 2008, 112, 3432-3440.

31 P. G. Bolhuis, D. Chandler, C. Dellago and P. L. Geissler, Annu. Rev. Phys. Chem., 2002, 53, 291-318.

32 J. Comer, F. Dehez, W. Cai and C. Chipot, J. Phys. Chem. C, 2013, 117, 26797-26803.

33 D. Branduardi, F. L. Gervasio and M. Parrinello, J. Chem. Phys., 2007, 126, 054103. 